\title{
Optical Power Allocation for Adaptive WDM Transmissions in Free Space Optical Networks
}

\author{
Hui Zhou, Shiwen Mao, and Prathima Agrawal \\ Department of Electrical and Computer Engineering, Auburn University, Auburn, AL \\ Email: \{hzz0016, agrawpr\}@auburn.edu, smao@ieee.org
}

\begin{abstract}
Attracting increasing attention in recent years, the Free Space Optics (FSO) technology has been recognized as a cost-effective wireless access technology for multi-Gigabit rate wireless networks. Radio over Free Space Optics (RoFSO) provides a promising enhancement to optical fiber systems. In an RoFSO system using wavelength-division multiplexing (WDM), it is possible to concurrently transmit multiple data streams consisting of various wireless services at very high rate. In this paper, we develop power allocation schemes for adaptive WDM transmissions to combat the effect of weather turbulence in FSO networks. The problem of optical power allocation under power budget and eye safety constraints is investigated for adaptive WDM transmission in RoFSO networks. Simulation results show that WDM RoFSO can support high data rates even over long distance or under bad weather conditions with an adequate system design.
\end{abstract}

\section{INTRODUCTION}

Recently, demand for multimedia service with high quality of service (QoS) requirements has been drastically increasing. To cater to the increasing demand, optical fibers have been utilized for years to deliver high volume of data. Due to the relatively high cost of deploying optical fibers, Free Space Optics (FSO) has been developed as a cost-effective wireless access technology for multi-Gigabit rate communication networks. FSO provides an excellent alternative to optical fiber systems for last-mile applications [1], ranging from local area network (LAN)-to-LAN connection for enterprise/campus, high capacity military communications, to disaster recovery and emergency response, among others.

Attracting considerable attention from the research community, FSO has also been studied for carrying various wireless services, as known as Radio on Free Space Optics (RoFSO). FSO systems can operate on wavelengths in the 1520-1600 nm range, which makes the development of wavelength-division multiplexing (WDM) FSO systems feasible. Advanced Dense Wavelength Division Multiplexing (DWDM) RoFSO systems are emerging to support the simultaneous transmission of multiple wireless signals [1]. Despite of its great potential of supporting data intensive communications, a line-of-sight (LOS) path is required in any FSO system. Consequently, FSO is highly susceptible to the atmospheric environment due to the inhomogeneity of air temperature and pressure, or flying objects [2]. To harvest the high potential of FSO, fading-mitigation techniques should be employed to mitigate atmospheric turbulence-induced intensity fluctuations.

To this end, topology control [2]-[5], load-balancing [6] and spatial diversity techniques [7] have been studied and proved to be effective in maintaining system performance.
Adaptive transmissions have been recently introduced into FSO systems and is emerging as a potential solution to mitigate the effect of atmospheric turbulence [8]. In FSO systems, channels are usually slow-fading and FSO transceivers have full-duplex capabilities. With negligible effect on data rates, a small portion of the bandwidth can be used for feedback of channel state information (CSI). In some hybrid RF/FSO systems, the RF channel can be used for CSI feedback. Thus reliable CSI could be available in FSO systems, which will be highly useful for designing adaptive transmission schemes.

In this paper, we propose optical power allocation schemes for an adaptive WDM RoFSO system in which variable wavelengths are adopted to mitigate the effect of weather turbulence. Proposed optical power allocation schemes optimally allocate transmit power to achieve maximum capacity and enhance the performance of the WDM system. Nowadays, the 1520-1600 $\mathrm{nm}$ wavelength band has already been used in FSO systems. Furthermore, the emerging quantum cascade laser (QCL) technology can offer great flexibility on adjusting an FSO transceiver to operate on the optimal transmit wavelengths [9]. Under a total power constraint, different optical powers can be allocated to the chosen wavelengths in a WDM RoFSO system, to achieve further enhanced system performance. We investigate the problem of optical power allocation under the power budget and eye safety power constraints for adaptive WDM transmission in RoFSO systems. To achieve capacity gain, we first analyze a conventional FSO system and develop a simple water-filling based algorithm to derive the optimal power allocation for the chosen wavelengths. For WDM RoFSO systems, a near-optimal RoFSO power allocation algorithm is developed based on the reformulationlinearization technique (RLT) [10], which can provide a linear programming (LP) relaxation of the complex problem. A computationally efficient scheme is also developed based on an approximation of the channel model. Finally, the performance of the proposed schemes are evaluated with simulations, and are demonstrated to be highly effective for achieving high system capacity under various scenarios.

The remainder of this paper is organized as follows. The related work is discussed in Section II and the system model is presented in Section III. The three power allocation schemes are developed in Section IV to fully utilize DWDM FSO systems. Simulation studies are presented in Section V. Section VI concludes this paper.

\section{RELATED WORK}

Attracting significant interest both in academia and industry, the FSO technology has been recognized as a promising 
solution for high capacity, long distance communications. RoFSO technology makes it possible to transmit multiple RF signals using WDM. WDM has been employed in FSO transmission systems and has been shown to be capable of supporting very high data rate transmission in [11]. RoFSO provides a promising alternative to optical fiber systems. In [12], the authors designed and evaluated an RoFSO system as an universal platform for the integration of optical fiber and FSO networks. In [13], optical fading in FSO Channels was statistically analyzed, while [1] provides a comprehensive study of RoFSO and the satisfactory results confirmed that the effect of scintillation on RoFSO performance can be estimated by an analytical model.

To mitigate weather turbulence, many research work has been done on developing adaptive transmission technology in FSO systems. Djordjevic in [14] applied the conventional wireless adaptive modulation and coding method in an FSO system and further studied adaptive low-density-parity-check (LDPC) coded modulation to compensate performance degradation when turbulence is strong. Karimi and Uysal in [8] designed transmission algorithms with consideration of the number of bits carried per chip time (BpC) in an FSO link, in which intensity modulation/direct detection (IM/DD) with $\mathrm{M}$-ary pulse position modulation (M-PPM) was employed.

Several other adaptive schemes have also been proposed and studied. In [9], the authors proposed using variable wavelength to combat the effects of atmospheric interference. Varying wavelength becomes feasible as the QCL technology becomes more mature. In [15], the authors proposed an adaptive transmission scheme to satisfy the requirements of various wireless services. A WDM power allocation method considering Optical Modulation Index(OMI) was proposed in their adaptive RoFSO system design. Authors in [16] studied the potential of multiple-input/multiple-output channel for combating link fading. However, FSO MIMO system performance is limited by thermally noise limited receivers and thus Avalanche photodiodes (APDs) [17] were studied and commonly used in FSO systems.

In this paper, we propose power allocation schemes for adaptive WDM transmissions to achieve capacity gain.In this adaptive WDM RoFSO system, variable number of wavelengths are adopted to mitigate weather turbulence and optical transmit power are optimally allocated to achieve maximum capacity gain. Although turbulence may not change significantly with wavelength in some weather conditions, considering the huge bandwidth that DWDM RoFSO system can support, it is still non-trivial to study the problem of utilizing wavelength properly. Moreover, Since QCL technology can offer great flexibility on adjusting wavelengths, more wavelengths can be utilized as FSO systems advance.

\section{System AND ChanNEL MOdEL}

\section{A. Channel Model}

FSO transceivers are highly directional, but FSO links are prone to degradation due to weather turbulence. We consider both effects of path loss and turbulence-induced fading over FSO links [18]. The optical channel state $h$ is modeled as a product of two factors:

$$
h=h_{l} h_{f},
$$

where $h_{l}$ denotes the attenuation and $h_{f}$ represents the atmospheric turbulence. $h_{l}$ is a function of optical wavelength $\Lambda$ and link length $d$, as

$$
h_{l}=\frac{A_{T X} A_{R X} e^{-\alpha d}}{(\Lambda d)^{2}},
$$

where $A_{T X}$ and $A_{R X}$ are the aperture areas of transmitter and receiver, respectively. The atmospheric attention coefficient $\alpha$ is give by

$$
\alpha=(3.91 / V)(\Lambda / 550)^{-q},
$$

where $V$ is the visibility in kilometers and $q$ is a parameter related to the visibility as [8]

$$
q= \begin{cases}0.585 V^{1 / 3}, & V \leq 6 \mathrm{~km} \\ 1.3, & 6 \mathrm{~km} \leq V \leq 50 \mathrm{~km}\end{cases}
$$

For modeling $h_{f}$, we assume atmospheric turbulence modeled as a Log-normal distribution [19]. The Log-normal model is a widely used fading model, especially under weak-tomoderate turbulence conditions.

The channel model for an FSO link can be written as

$$
y=h \cdot P_{t} \cdot x+n,
$$

where $x$ and $y$ are transmitted and received signal respectively; $n$ is the additive Gaussian noise; $P_{t}$ is the power of the transmitted pulse.

\section{B. System Model}

Radio on Free Space Optics (RoFSO) is a new technology that provides high data rate and reliable transmission. The RoFSO system using WDM allows simultaneous transmission of multiple data streams consisting of various wireless services at very high rates.

We assume a WDM FSO system that is capable of operating from the $1520 \mathrm{~nm}$ wavelength band to the $1600 \mathrm{~nm}$ wavelength band. Apart from the data-transmitting antenna, we assume that an atmospheric influence measurement antenna or weather measurement device is equipped at the FSO BS. Thus, we can estimate atmospheric loss for channels using different wavelengths. Alternatively, CSI can be obtained by using a small portion of the bandwidth to provide channel information without affecting data rates. In some hybrid RF/FSO systems, the RF channel can be used for CSI feedback. Since atmospheric turbulence is a major degrading factor in FSO systems, we propose power allocation schemes for the adaptive FSO system, in which both wavelength and transmit power will be adaptively allocated according to channel conditions.

An important parameter to evaluate the performance of RFFSO is the carrier to noise ratio (CNR). The CNR of an RoFSO system using an APD photo detector is given as [1]

$$
C N R=\frac{0.5\left(O M I \cdot m P_{r}\right)^{2}}{R I N \cdot P_{r}^{2}+2 e m^{(2+F)} P_{r}+4 K T \cdot G_{f}},
$$

where OMI is the optical modulation index, $m$ is the photodiode gain, $T$ is the temperature, $K$ is the Boltzman's constant, $e$ is the electrical charge and $G_{f}$ is the photodiode output conductance. In the denominator, $4 K T G_{f}$ is thermal noise; $2 e m^{(2+F)} P_{r}$ is optical short noise, and $R I N P_{r}^{2}$ is the 
relative intensity noise from Laser diode(LD). The numerator represents the received signal power. $P_{r}=r P_{p d}$, where $r$ is the photodiode responsivity and $P_{p d}$ is the received power at the detector and is given as the product of transmit power and channel gain. Without loss of generality, we assume all tones are modulated with the same OMI that will not introduce intermodulation distortion.

\section{ADAPTIVE WDM TRANSMISSION}

To mitigate the effect of weather turbulence, we adapt wavelength and adjust power allocation to achieve better system performance. First, our proposed FSO system uses wavelength $\Lambda \in[1520,1600] \mathrm{nm}$ and the available wavelengths are divided into $N$ parts, non-overlapping with adequate spacing. We assume the FSO channel is slow varying [8]. For each channel with wavelength $\Lambda_{i}$, we can estimate its channel state $h_{i}$ or get feedback channel information from feedback channel.

In different weather conditions, it is desirable to choose the wavelengths that have the best channel conditions. We assume that the WDM FSO system will use at most $M$ different wavelengths. Among the $N$ available wavelengths, we will first choose $M$ wavelengths that have the greatest channel gains. The next step is to allocate transmit powers to different wavelengths such that the system capacity is maximized.

\section{A. Conventional FSO System}

We first consider conventional modeling of wireless channels under white Gaussian noise. For the sake of simplicity, we denote channel gain for channel $i$ by

$$
h_{i}=\frac{\left|h_{l} h_{f}\right|^{2}}{N_{0}} \text {. }
$$

According to the estimated channel gains, we choose $M$ wavelengths that can offer the greatest channel gains from the $N$ available wavelengths.

Let $P_{i}$ be the optical power allocated to channel $i$. Due to fixed power budget $P_{\max }$ for each FSO base station, we have the following total power constraint

$$
\sum_{i=1}^{M} P_{i} \leq P_{\max }
$$

In FSO systems, eye safety problem should be always taken into consideration in the system design. Thus we have the additional power constraint

$$
0 \leq P_{i} \leq \bar{P} \text {. }
$$

where $\bar{P}$ is the peak power bound for the transmit powers. Thus, we formulate the following capacity maximization problem.

$$
\begin{aligned}
\max & \sum_{i=1}^{M} \log \left(1+P_{i} h_{i}\right) \\
\text { s.t. } & \sum_{i=1}^{M} P_{i} \leq P_{\max } \\
& 0 \leq P_{i} \leq \bar{P}, \forall i .
\end{aligned}
$$

By applying Karush-Kuhn-Tucker(KKT) theorem, we can find that optimal power allocation satisfies

$$
\left\{\begin{array}{l}
P_{i}=\min \left\{\bar{P},\left[\frac{1}{\lambda}-\frac{1}{h_{i}}\right]^{+}\right\} \\
\sum_{i=1}^{M} P_{i}=P_{\max }
\end{array}\right.
$$

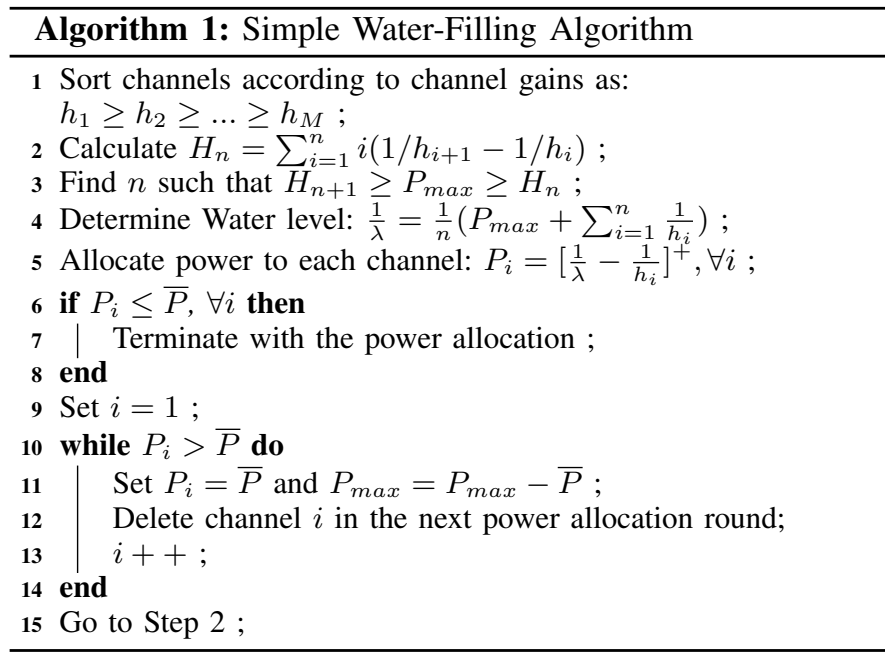

where $\lambda$ is the Lagrange multiplier. The inverse of $\lambda$ is often regarded as the water level.

The algorithm to solve the capacity maximization problem is to first sort the channels according to their channel gains. We then find the number of channels $n$, which are allocated with a nonzero power, as in Steps 2-3. The water volume $H_{n}$ that is required to fill $n$ channels can be calculated as $\sum_{i=1}^{n} i\left(1 / h_{i+1}-1 / h_{i}\right)$ and $H_{n}$ should not be greater than $P_{\max }$. Then we can calculate the water level and allocate power to each selected channel according to (13). This procedure is base on the assumption that a feasible power should also satisfy the eye safety power constraint. If the allocated power $\frac{1}{\lambda}-\frac{1}{h_{i}}$ is greater than $\bar{P}$, we need to adjust the water level accordingly. The detailed water-filling algorithm is presented in Algorithm 1.

\section{B. DWDM RoFSO System}

Next, we develop the model for the RoFSO channels as described in Section III, which is more suitable for RoFSO using APD photo-detectors. CNR defined in Section III-B will be an important parameter to evaluate the RoFSO performance.

1) Reformulation and Relaxation Based Approach: To simply notation, we denote constant value $0.5(m O M I)^{2}$ by $a$, relative intensity noise level $R I N$ by $b$, optical short noise $2 e m^{(2+F)}$ by $c$, and thermal noise $4 K T G_{f}$ by $d$. Thus, our adaptive power allocation problem becomes

$$
\begin{aligned}
\max & \sum_{i=1}^{M} \log \left(1+\frac{a_{i}\left(P_{i} h_{i}\right)^{2}}{b_{i}\left(P_{i} h_{i}\right)^{2}+c_{i} P_{i} h_{i}+d_{i}}\right) \\
\text { s.t. } & \sum_{i=1}^{M} P_{i} \leq P_{\max } \\
& 0 \leq P_{i} \leq \bar{P}, \forall i .
\end{aligned}
$$

If we denote CNR by

$$
\gamma_{i}=\frac{a_{i}\left(P_{i} h_{i}\right)^{2}}{b_{i}\left(P_{i} h_{i}\right)^{2}+c_{i} P_{i} h_{i}+d_{i}},
$$


the optimization problem, termed Problem OPT-FSO, can be rewritten as

$$
\begin{array}{ll}
\max & \sum_{i=1}^{M} \log \left(1+\gamma_{i}\right) \\
\text { s.t. } & \sum_{i=1}^{M} P_{i} \leq P_{\max } \\
& 0 \leq P_{i} \leq \bar{P}, \forall i \\
& \gamma_{i}=\frac{a_{i}\left(P_{i} h_{i}\right)^{2}}{b_{i}\left(P_{i} h_{i}\right)^{2}+c_{i} P_{i} h_{i}+d_{i}}, \forall i .
\end{array}
$$

It is challenging to solve this problem due to its complexity and nonlinear nonconvex properties. In the following, we adopt the RLT technique to obtain an LP relaxation of Problem OPTFSO and derive a feasible near-optimal solution.

The RLT relaxation is as follows. Letting $c_{i}=\log \left(1+\gamma_{i}\right)$, the objective function $\sum_{i=1}^{M} c_{i}$ will now be linear and new constraints $c_{i}=\log \left(1+\gamma_{i}\right)$ are introduced.We first linearize the logarithmic terms in the new constraints using the polyhedral outer approximation as follows.

Letting $P_{i}$ be 0 and $\bar{P}$, we obtain the lower and upper bounds of $\gamma_{i}$, respectively. We denote the upper bound of $\gamma_{i}$ by $\bar{\gamma}_{i}$. We use the four-point approximation and obtain the following new linear constraints.

$$
\left\{\begin{array}{l}
c_{i} \geq \frac{\gamma_{i} \cdot \log \left(1+\bar{\gamma}_{i}\right)}{\bar{\gamma}_{i}} \\
c_{i} \leq \log \left(1+\gamma_{i}^{k}\right)+\frac{\gamma_{i}-\gamma_{i}^{k}}{1+\gamma_{i}^{k}}
\end{array}\right.
$$

where $\gamma_{i}^{k}=\frac{k \cdot \bar{\gamma}_{i}}{3}$, for $k=0,1,2,3$. The first equation in (22) is for the segment connecting the two end points of the logarithm function, and the second equation in (22) is for the tangent lines at the four points on the logarithm function.

Problem OPT-FSO now becomes a polynomial programming problem. We next introduce substitution variables and the corresponding RLT bound-factor product constraints to remove the quadratic terms and to obtain an LP relaxation. Specifically, constraint (21) contains quadratic terms. We can rewrite (21) as

$$
b_{i}\left(P_{i} h_{i}\right)^{2} \gamma_{i}+c_{i} h_{i} P_{i} \gamma_{i}+d_{i} \gamma_{i}-a_{i}\left(P_{i} h_{i}\right)^{2}=0 .
$$

To remove quadratic terms, we define substitution variables $u_{i}=P_{i}^{2}, v_{i}=\gamma_{i} P_{i}$, and $w_{i}=\gamma_{i} u_{i}$, for all $i$. Thus constraint (21) becomes

$$
b_{i} h_{i}^{2} w_{i}+c_{i} h_{i} v_{i}+d_{i} \gamma_{i}-a_{i} h_{i}^{2} u_{i}=0 .
$$

Since $P_{i}$ is bounded, it can easily show that $u_{i} \in\left(0, \bar{P}^{2}\right)$. For variables $v_{i}$, we can obtain the following RLT bound-factor product constraints.

$$
\left\{\begin{array}{l}
\left(\gamma_{i}-0\right)\left(P_{i}-0\right) \geq 0 \\
\left(\bar{\gamma}_{i}-\gamma_{i}\right)\left(P_{i}-0\right) \geq 0 \\
\left(\gamma_{i}-0\right)\left(\bar{P}-P_{i}\right) \geq 0 \\
\left(\bar{\gamma}_{i}-\gamma_{i}\right)\left(\bar{P}-P_{i}\right) \geq 0
\end{array}\right.
$$

Substituting $v_{i}=\gamma_{i} P_{i}$, we obtain the following four linear constraints for variable $v_{i}$.

$$
\left\{\begin{array}{l}
v_{i} \geq 0 \\
\bar{\gamma}_{i} P_{i}-v_{i} \geq 0 \\
\bar{P} \gamma_{i}-v_{i} \geq 0 \\
\bar{P} \bar{\gamma}_{i}-\bar{\gamma}_{i} P_{i}-\bar{P} \gamma_{i}+v_{i} \geq 0
\end{array}\right.
$$

We deal with the variables $w_{i}$ in the same manner and obtain the following four linear constraints for variable $w_{i}$.

$$
\left\{\begin{array}{l}
w_{i} \geq 0 \\
\bar{\gamma}_{i} u_{i}-w_{i} \geq 0 \\
\bar{P}^{2} \gamma_{i}-w_{i} \geq 0 \\
\bar{P}^{2} \bar{\gamma}_{i}-\bar{\gamma}_{i} u_{i}-\bar{P}^{2} \gamma_{i}+w_{i} \geq 0
\end{array}\right.
$$

Now the original problem is relaxed to an LP problem with the additional constraints and variables, which can be solved in polynomial time with an LP solver. Note that during the procedure of reformulation and linearization, we preserve the original power constraints of Problem OPT-FSO, i.e., (19) and (20). Hence the optimal transmit power allocation policy obtained for the LP relaxation is also feasible to the original problem OPT-FSO. The feasibility of the LP solution is summarized in the following proposition.

Proposition 1. The optimal transmit power allocation policy to the LP relaxation of Problem OPT-FSO is a feasible solution to the original problem.

2) Water-filling Based Approach: Due to the complexity of the RLT-based method, it may not be suitable when the channels vary quickly. We also develop a more computational cost-effective scheme in the following. If we ignore the relative intensity noise and optical short noise, CNR can be approximated by $0.5 P_{r}^{2} \frac{(m O M I)^{2}}{4 K T G_{f}}$. To simplify notation, denote constant value $0.5(m O M I)^{2} / 4 K T G_{f}$ by $a$. We obtain the following optimization problem.

$$
\begin{aligned}
\max & \sum_{i=1}^{M} \log \left(1+a_{i}\left(P_{i} h_{i}\right)^{2}\right) \\
\text { s.t. } & \sum_{i=1}^{M} P_{i} \leq P_{\max } \\
& 0 \leq P_{i} \leq \bar{P}, \forall i .
\end{aligned}
$$

According to Karush-Kuhn-Tucker(KKT) theorem, if $\boldsymbol{P}^{\star}=\left[P_{1}^{\star}, P_{2}^{\star}, \cdots, P_{M}^{\star}\right]$ is a local maximizer for the above optimization problem, there exists $\lambda \in \mathbb{R}$ and $\lambda_{i} \in \mathbb{R}$, for $i \in\{1, \cdots, M\}$, such that

$$
\left\{\begin{array}{l}
\frac{\partial\left[\log \left(1+a_{i}\left(P_{i}^{\star} h_{i}\right)^{2}\right)\right]}{\partial P_{i}}-\lambda-\lambda_{i}=0, \forall i \\
\lambda\left(\sum_{i=1}^{M} P_{i}^{\star}-P_{\max }\right)=0 \\
\lambda_{i}\left(P_{i}^{\star}-\bar{P}\right)=0, \forall i \\
\lambda \geq 0, \lambda_{i} \geq 0, \forall i
\end{array}\right.
$$

According to (31), if $\lambda_{i}>0$, then $P_{i}^{\star}=\bar{P}$; and if $\lambda_{i}=0$, we can solve (31) to have

$$
P_{i}^{\star}=\frac{1}{\lambda}+\sqrt{\frac{1}{\lambda^{2}}-\frac{1}{a_{i} h_{i}^{2}}} .
$$

Thus we find that the optimal power allocation satisfies

$$
\left\{\begin{array}{l}
P_{i}=\min \left\{\bar{P}, \frac{1}{\lambda}+\sqrt{\frac{1}{\lambda^{2}}-\frac{1}{a_{i} h_{i}^{2}}}\right\}, \text { if } \lambda^{2} \leq a_{i} h_{i}^{2} \\
P_{i}=0, \text { otherwise } \\
\sum_{i=1}^{M} P_{i}=P_{\max } .
\end{array}\right.
$$

Regarding the inverse of $\lambda$ as some kind of "water level," we find that the greater the channel gain, the larger the 


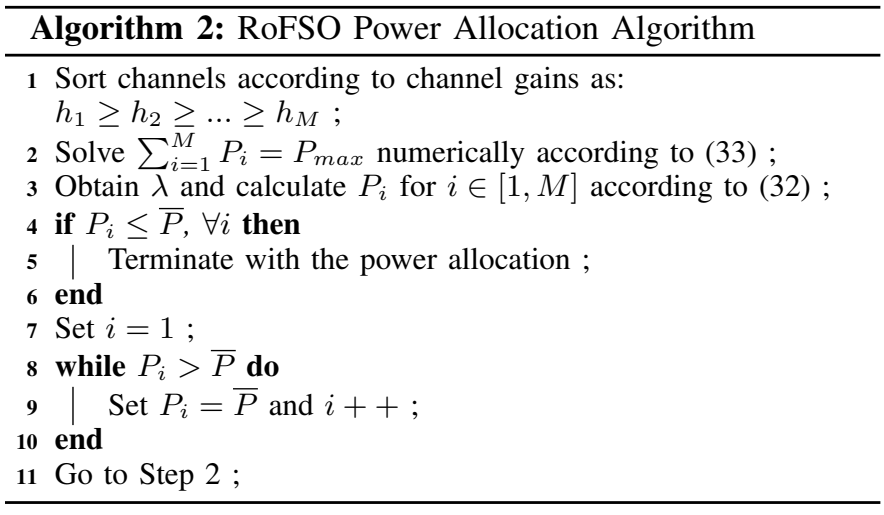

TABLE I. Simulation Parameters

\begin{tabular}{l|l|l}
\hline Symbol & Value & Definition \\
\hline$D_{t}$ & $15 \mathrm{~mm}$ & Tx. Aperture Diameter \\
$D_{r}$ & $0.1 \mathrm{~m}$ & Rx. Aperture Diameter \\
$B$ & $1 \mathrm{GHz}$ & Bandwidth \\
$d$ & $1 \mathrm{Km}$ & Distance \\
$P_{\max }$ & $0.5 \mathrm{~W}$ & Power budget \\
$P$ & $0.1 \mathrm{~W}$ & Peak power constraint \\
$O M I$ & $17.5 \%$ & Optical modulation index \\
$m$ & 5 & Photodiode gain \\
$R I N$ & $-150 \mathrm{~dB} / \mathrm{Hz}$ & Relatively intensity noise \\
\hline
\end{tabular}

deviation of the power allocated to this channel from the water level. Usually we cannot directly solve from (33) the optimal power allocation. An iterative algorithms is needed to obtain an appropriate $\lambda$ and solve this optimization problem. The detailed algorithm is presented in Algorithm 2.

\section{Performance Evaluation}

In this section, we evaluate the performance of the proposed algorithms with MATLAB simulations. We calculate channel gains as shown in Section III-A and CNR are calculated according to (6) for the evaluated schemes. The simulation parameters are the same from prior work and are listed in Table I [1] [15]. We investigate the three algorithms introduced in the previous section for power allocation in WDM RoFSO system. Wavelengths in the band $1520-1580 \mathrm{~nm}$ are assumed in our WDM RoFSO simulations. Adequate guard band between adjacent wavelengths is needed; according to [11], we adopt a $5 \mathrm{~nm}$ guard band between adjacent wavelengths used in the simulations.

In Fig. 1, we compare the three algorithms introduced in Section IV and examine the impact of the power budget on the total system capacity. We increase $P_{\max }$ from 0.5 to 1 with step-size 0.1 and plot the total capacity. As can be seen in Fig. 1, the RoFSO power allocation algorithm outperforms the other two algorithms with considerable gains. Since the relative intensity noise and optical short noise are very small in our simulations, the RoFSO power allocation algorithm will achieve the best near-optimal solution. The RLT algorithm, although consumes much running time, can only produce an optimal power allocation for the relaxed LP problem. The power allocation solution obtained from RLT is feasible but achieves the worst performance in terms of capacity gain due to relaxation. We also find that the total capacity increases along with the power budget for both the water-filling algorithm and

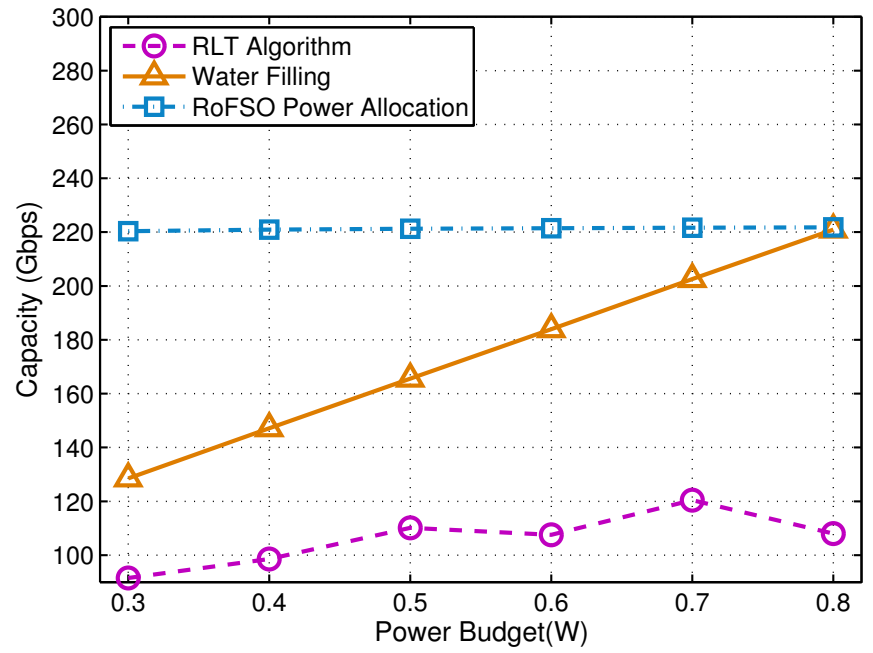

Fig. 1. System capacity vs. the power budget $P_{\max }$.

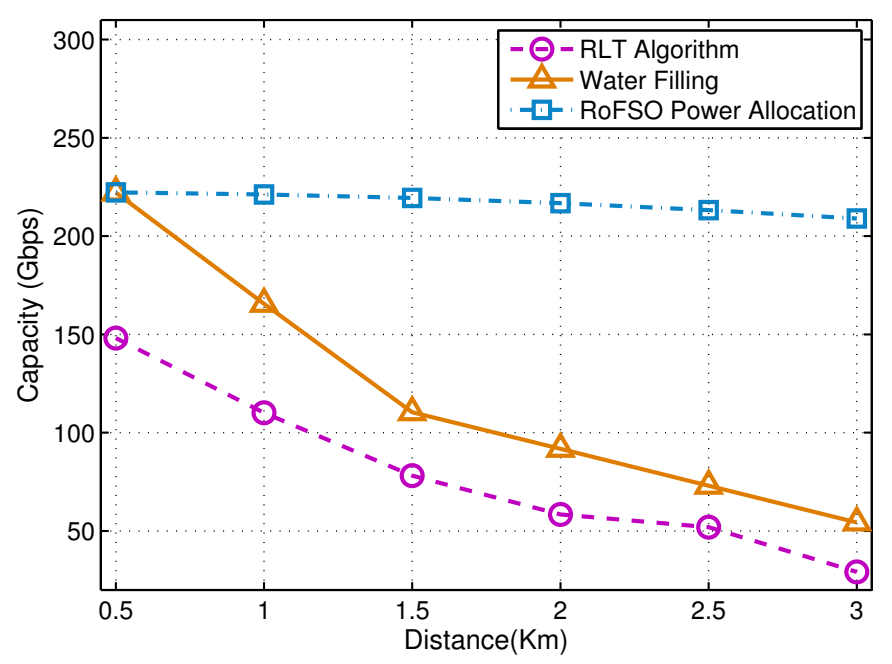

Fig. 2. System capacity vs. the distance.

RoFSO power allocation algorithm, albeit not obviously for the latter. After the power budget becomes even larger, there is much less space for capacity increment due to the eye safety power constraint.

We also plot the total capacity vs. the distance between the FSO transceivers in Fig. 2. As expected, the total capacity decrease as the distance is increased. The capacity obtained by using the RoFSO power allocation algorithm, however, decreases most slowly and is always greater than capacities produced by the other two schemes. These simulation results indicate that with proper system design, the RoFSO system can support high data rates even over long distance and under bad weather conditions.

\section{CONCLUSIONS}

In this paper, we investigated the problem of optical power allocation under a power budget constraint and eye safety power constraint for adaptive WDM transmission to mitigate the effect of weather turbulence, and solution algorithms are developed. For convectional transmission systems, a simple 
water-filling algorithm can be adopted to allocate power to different wavelengths; for WDM RoFSO systems, a RoFSO power allocation algorithm was demonstrated to achieve the greatest system capacity. It is capable of supporting high data rates even over long distance or under bad weather conditions.

\section{ACKNOWLEDGMENT}

This work is supported in part by the U.S. National Science Foundation (NSF) under Grant CNS-1145446, and through the NSF Broadband Wireless Access \& Applications Center (BWAC) at Auburn University. Any opinions, findings, and conclusions or recommendations expressed in this material are those of the author(s) and do not necessarily reflect the views of the NSF.

\section{REFERENCES}

[1] P. Dat, A. Bekkali, K. Kazaura, K. Wakamori et al., "Studies on characterizing the transmission of RF signals over a turbulent FSO link," Optics Express, vol. 17, pp. 7731-7743, 2009.

[2] H. Zhou, A. Babaei, S. Mao, and P. Agrawal, "Algebraic connectivity of degree constrained spanning trees for FSO networks," in Proc. IEEE ICC'13, Budapest, Hungary, June 2013, pp. 1-6.

[3] I. K. Son, S. Kim, and S. Mao, "Building robust spanning trees in free space optical networks," in IEEE MILCOM'10, San Jose, CA, 2010, pp. $1857-1862$.

[4] I. K. Son and S. Mao, "Design and optimization of a tiered wireless access network," in Proc. IEEE INFOCOM'10, San Diego, CA, Mar 2010, pp. 1-9.

[5] I.-K. Son, S. Mao, and S. K. Das, "On the design and optimization of a free space optical access network," Elsevier Optical Switching and Networking, vol. 11, no. Part.A, pp. 29-43, Jan. 2014.

[6] - "On joint topology design and load balancing in fso networks," Elsevier Optical Switching and Networking, vol. 11, no. Part A, pp. 92-104, Jan. 2014

[7] A. Farid and S. Hranilovic, "Diversity gain and outage probability for MIMO free-space optical links with misalignment," IEEE Trans. Commun., vol. 60, no. 2, pp. 479-487, Feb. 2012.

[8] M. Karimi and M. Uysal, "Novel adaptive transmission algorithms for free-space optical links," IEEE Trans. Commun., vol. 60, no. 12, pp. 3808-3815, Dec. 2012.

[9] X. Liu, "Free-space optics optimization models for building sway and atmospheric interference using variable wavelength," IEEE Trans. Commun., vol. 57, no. 2, pp. 492-498, Feb. 2009.

[10] Y. Huang and S. Mao, "Downlink power control for variable bit rate videos over multicell wireless networks," in Proc. IEEE INFOCOM'11, Apr. 2011, pp. 2561-2569.

[11] E. Ciaramella, Y. Arimoto, G. Contestabile, M. Presi, A. D'Errico, V. Guarino, and M. Matsumoto, "1.28 terabit/s (32x40 gbit/s) WDM transmission system for free space optical communications," IEEE $J$. Sel. Areas Commun., vol. 27, no. 9, pp. 1639-1645, Dec. 2009.

[12] K. Kazaura, K. Wakamori, M. Matsumoto, T. Higashino, K. Tsukamoto, and S. Komaki, "RoFSO: A universal platform for convergence of fiber and free-space optical communication networks," in Innovations for Digital Inclusions, 2009. ITU-T Kaleidoscope:, 2009, pp. 1-8.

[13] K.-H. Kim, T. Higashino, K. Tsukamoto et al., "Statistical analysis on the optical fading in free space optical channel for RoFSO link design," in Proc. SPIE 7620, Broadband Access Communication Technologies IV, 76200G, 2010, pp. 1-10.

[14] I. Djordjevic, "Adaptive modulation and coding for free-space optical channels," IEEE/OSA J. Opt. Commun. Netw., vol. 2, no. 5, pp. 221229, May 2010.

[15] K.-H. Kim, T. Higashino, K. Tsukamoto, and S. Komaki, "WDM optical power allocation method for adaptive radio on free space optics system design," in 2011 International Topical Meeting on Microwave Photonics \& 2011 Asia-Pacific Microwave Photonics Conference (MWP/APMP), Singapore, Oct. 2011, pp. 361-364.
[16] S. Wilson, M. Brandt-Pearce, Q. Cao, and I. Leveque, J.H., "Free-space optical mimo transmission with q-ary ppm," Communications, IEEE Transactions on, vol. 53, no. 8, pp. 1402-1412, 2005.

[17] N. Cvijetic, S. Wilson, and M. Brandt-Pearce, "Performance bounds for free-space optical mimo systems with apd receivers in atmospheric turbulence," Selected Areas in Communications, IEEE Journal on, vol. 26, no. 3, pp. 3-12, 2008.

[18] H. Zhou, D. Hu, S. Mao, and P. Agrawal, "Joint relay selection and power allocation in cooperative FSO networks," in Proc. IEEE GLOBECOM'13, Atlanta, GA, Dec. 2013, pp. 1-6.

[19] M. Safari, M. Rad, and M. Uysal, "Multi-hop relaying over the atmospheric poisson channel: Outage analysis and optimization," IEEE Trans. Commun., vol. 60, no. 3, pp. 817-829, Mar. 2012. 